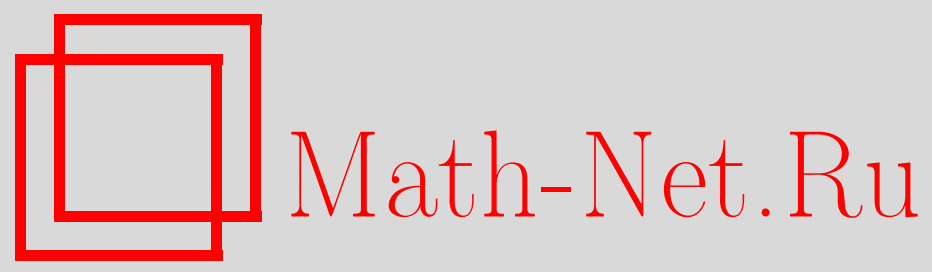

И. А. Чельцов, Ограниченность трехмерных многообразий Фано целого индекса, Матем. заметки, 1999, том 66, выпуск $3,445-451$

DOI: https://doi.org/10.4213/mzm1186

Использование Общероссийского математического портала Math-Net.Ru подразумевает, что вы прочитали и согласны с пользовательским соглашением http://www . mathnet.ru/rus/agreement

Параметры загрузки:

IP : 54.162 .27 .143

26 апреля 2023 г., 18:20:46

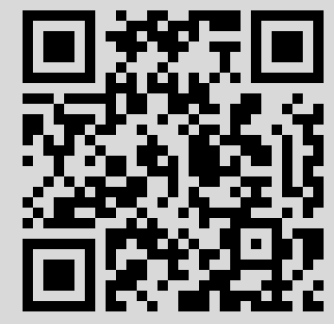




\title{
ОГРАНИЧЕННОСТЬ ТРЕХМЕРНЫХ МНОГООБРАЗИЙ ФАНО ЦЕЛОГО ИНДЕКСА
}

\author{
И. А. Чельцов
}

В данной работе эффективно ограничивается куб антиканонического класса трехмерного многообразия Фано с каноническими особенностями и целым индексом Фано.

Библиография: 16 названий.

Все рассматриваемые многообразия комплексные и проективные. Основные определения, обозначения и понятия содержатся в [1] и [2]. Автор очень благодарен В.А. Исковских, В. Клейнерту, Ю.Г. Прохорову и В.В. Шокурову за полезные беседы и внимание, а также Университету Джона Хопкинса (США) и Университету Хумбольда в Берлине (Германия) за гостеприимство.

1. Введение. Основная цель данной статьи состоит в доказательстве следующего результата.

Теорема 1. Пусть $X$ есть трехмерное многообразие Фано с каноническими особенностями $u-K_{X} \sim_{\mathbb{Q}} H$, где $H$ это обильный дивизор Картье. Тогда $H^{3} \leqslant$ 184/I, әде I есть индекс горенштейневости $X$.

ЗАмечаниЕ 1. В теореме $1 I=1$ или 2 (см. [3]). Рассматривая глобальное каноническое накрытие $X$ (см. [4]), далее везде можно считать, что $I=1$, т.е. $X$ горенштейнево. Легко видеть, что тогда $-K_{X} \sim H$.

ЗАмечАниЕ 2. Оценка $H^{3}$ в теореме 1 , по-видимому, далека от совершенства. В случае когда $X$ гладко, $H^{3} \leqslant 64$ (см. [2]), причем равенство достигается для $\mathbb{P}^{3}$. В случае когда $X$ имеет терминальные горенштейневые особенности, $X$ можно продеформировать в гладкое (см. [5]) и, таким образом, $H^{3} \leqslant 64$. В случае когда $X$ имеет терминальные особенности, из рассмотрения канонического накрытия $X$ (см. замечание 1 ) следует, что $H^{3} \leqslant 64 / I$.

Существуют примеры трехмерных многообразий Фано с каноническими горенштейневыми особенностями и $H^{3}=72$, например конус над антиканонически вложенной поверхностью Дель Пещцо степени 9 (см. [2]).

В случае когда $X$ имеет негоренштейневые терминальные фактор-особенности, из классификации следует, что $H^{3} \leqslant 24$, причем равенство достигается для фактора $\mathbb{P}^{1} \times$ $\mathbb{P}^{1} \times \mathbb{P}^{1}$ по инволюции, имеющей конечное число неподвижных точек (см. [4]).

В [6] и [7] Фано исследовал трехмерные многообразия с гиперплоскими сечениями поверхностями типа $K 3$ и Энриквеса (с каноническими особенностями) соответственно. 
Допуская нормальность таких многообразий, можно показать, что это в точности многообразия из теоремы 1 с очень обильным дивизором $H$, причем $I=1$ в случае когда $H$ есть поверхность типа $K 3$ и $I=2$ в случае когда $H$ есть поверхность Энриквеса (см. [3]). Интересно отметить, что Фано гипотетически утверждал, что $H^{3} \leqslant 72$ в горенштейневом случае и $H^{3} \leqslant 24$ в негоренштейневом.

2. $X$ c $\mathrm{Bs}|H| \neq \varnothing$.

Теорема 2. Если в теореме $1 \quad I=1$ и $\mathrm{Bs}|H| \neq \varnothing$, mo $H^{3} \leqslant 46$.

ДокАЗАТЕЛЬСтво. Из [8] следует, что при $H^{3}>2 \mathrm{Bs}|H|$ есть гладкая рациональная кривая $Z \in X \backslash \operatorname{Sing}(X)$, и если $\pi: \bar{X} \rightarrow X$ есть раздутие $Z$, то следующая диаграмма коммутативна:

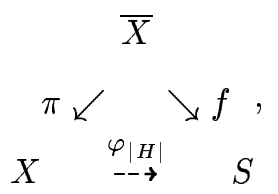

где морфизм $f$ есть расслоение на эллиптические кривые, $\pi^{-1}(Z)=\bar{Z}$ есть сечение $f$. Более того, $\left.f\right|_{\bar{Z}}: \bar{Z} \rightarrow S$ есть либо изоморфизм, либо стягивание исключительного сечения линейчатой поверхности $\bar{Z} \cong \mathbb{F}_{n}\left(n \in \mathbb{N}_{0}\right)$.

Пусть $\bar{E}$ и $\bar{F}$ - поверхности, заметаемые слоями морфизма $f$, проходящими слой и исключительное сечение линейчатой поверхности $\bar{Z}$ соответственно. Имеет место следующее равенство

$$
K_{\bar{X}}=-\bar{F}-\frac{m+n}{2} \bar{E}
$$

где $m=\left(H^{3}+2\right) / 2$, причем если $S$ особо, то $m=n$.

Если поверхность $S$ неособа, то Bs $|\bar{E}|=\varnothing$. Если поверхность $S$ особа и $\operatorname{Bs}|\bar{E}| \neq \varnothing$, то $\operatorname{dim}(\mathrm{Bs}|\bar{E}|)=0$, поскольку Bs $|\bar{E}| \cap \bar{Z}=\mathrm{Bs}|\bar{E}| \cap \pi^{-1}(H)=\varnothing$ и $H$ - обилен. Заметим, что $\mathrm{Bs}|\bar{E}| \neq \varnothing$, только если $\bar{E}$ не $\mathbb{Q}$-Картье дивизор. В последнем случае пусть $g: \bar{X}^{\prime} \rightarrow \bar{X}$ есть мальй морфизм такой, что $g^{-1}(\bar{E})$ есть $g$-обильный $\mathbb{Q}-$ Картье дивизор (см. [9]). Тогда Bs $\left|g^{-1}(\bar{E})\right|=\varnothing$. Действительно, если Bs $\left|g^{-1}(\bar{E})\right| \neq \varnothing$, то Bs $\left|g^{-1}(\bar{E})\right|=\bar{E}_{1}^{\prime} \cap \bar{E}_{2}^{\prime}$ для достаточно общих дивизоров $\bar{E}_{1}^{\prime}$ и $\bar{E}_{2}^{\prime}$ из линейной системы $\left|g^{-1}(\bar{E})\right|$ и $\mathrm{Bs}\left|g^{-1}(\bar{E})\right|$ лежит в слоях морфизма $\left.f \circ g\right|_{E_{1}^{\prime}}: \bar{E}_{1}^{\prime} \rightarrow \mathbb{P}^{1}$. Форма пересечения кривых в слоях морфизма $\left.f \circ g\right|_{E_{1}^{\prime}}$ полуотрищательно определена и, следовательно, $g^{-1}(\bar{E})^{3} \leqslant 0$, но $\bar{E}_{1}^{\prime} \cap \bar{E}_{2}^{\prime}$ лежит в слоях морфизма $g$ и по $g$-обильности дивизора $g^{-1}(\bar{E}) g^{-1}(\bar{E})^{3}>0$. Следовательно, $\mathrm{Bs}\left|g^{-1}(\bar{E})\right|=\varnothing$. Далее, заменяя, где нужно, $\bar{X}$ на $\bar{X}^{\prime}$, можно считать, что $\mathrm{Bs}|\bar{E}|=\varnothing$.

Утверждение теоремы следует из неравенства $(m+n) / 2 \leqslant 12$. Допустим, что последнее неверно. Рассмотрим лог-пару:

$$
\left(K_{\bar{X}}, \gamma\left(\bar{Z}+\bar{F}+\frac{m+n}{4} \bar{E}_{1}+\frac{m+n}{4} \bar{E}_{2}\right)\right),
$$

где $\gamma \in \mathbb{Q}_{>0}, \bar{E}_{1}$ и $\bar{E}_{2}-$ два достаточно общих дивизора из линейной системы $|\bar{E}|$. Для $\gamma<1$ хорошо известно, что множество лог-канонических особенностей (см. [10]) 
лог-пары $(*)$ связно. Действительно, рассмотрим лог-разрешение $\tau: Y \rightarrow \bar{X}$. Вьполнено следующее соотношение:

$$
K_{Y}+\tau^{*}\left(\pi^{*}((1-\gamma) H)\right) \equiv \tau^{*}\left(K_{\bar{X}}+\pi^{*}(H)\right)-\sum_{i=1}^{k} a_{i} E_{i}+\sum_{j=1}^{l} b_{j} D_{j}
$$

где $E_{i}$ и $D_{j}$ - неприводимые дивизоры на $Y, a_{i}$ и $b_{j}$ - положительные рациональные числа и дивизоры $D_{j}$ исключительны для морфизма $\tau$. Нужно показать связность множества $V=-\left\lceil-\sum_{i=1}^{k} a_{i} E_{i}\right\rceil$. Заметим, что

$$
\pi^{*}(H)=\bar{Z}+\bar{F}+\frac{m+n}{2} \bar{E} .
$$

В окрестности $\bar{Z}$ можно считать $\tau$ изоморфизмом и $V$ есть либо $\varnothing$, если

$$
\gamma<\frac{4}{m+n}
$$

либо $\left\{\bar{E}_{1}, \bar{E}_{2}\right\}$ в противном случае. По теореме об обращении в нуль Каваматы-Фивега (см. [1]):

$$
H^{1}\left(\tau^{*}\left(K_{\bar{X}}+\pi^{*}(H)\right)+\left\lceil-\sum_{i=1}^{k} a_{i} E_{i}\right\rceil+\left\lceil\sum_{j=1}^{l} b_{j} D_{j}\right\rceil\right)=0 .
$$

Последнее влечет сюръективность:

$$
H^{0}\left(\tau^{*}(\bar{Z})+\left\lceil\sum_{j=1}^{l} b_{j} D_{j}\right\rceil\right) \rightarrow H^{0}\left(\left.\left(\tau^{*}(\bar{Z})+\left\lceil\sum_{j=1}^{l} b_{j} D_{j}\right\rceil\right)\right|_{V}\right) \rightarrow 0
$$

но в силу $\tau$-исключительности дивизоров $D_{j}$ и неподвижности дивизора $\bar{Z}$ имеем:

$$
H^{0}\left(\tau^{*}(\bar{Z})+\left\lceil\sum_{j=1}^{l} b_{j} D_{j}\right\rceil\right)=\mathbb{C}
$$

Теперь покажем, что в предположении $(m+n) / 2>12$ для $\gamma=1 /(6+\varepsilon)(0<$ $\varepsilon \ll 1)$ множество лог-канонических особенностей лог-пары $(*)$ несвязно. Во-первых, в нем содержатся дивизоры $\bar{E}_{1}$ и $\bar{E}_{2}$. Во-вторых, в окрестности $\bar{Z}$ утверждение тривиально. Следовательно, достаточно показать, что множество лог-канонических особенностей лог-пары

$$
\left(K_{\bar{X}}, \frac{1}{6+\varepsilon} \bar{F}\right)
$$

не имеет элементов коразмерности два, не лежащих в слоях морфизма $f$. Легко видеть, что в коразмерности два особенности пары $(* *)$, не лежащие в слоях морфизма $f$, это в точности особенности лог-пары

$$
\left(K_{\bar{E}_{1}}, \frac{1}{6+\varepsilon} \bar{F} \cap \bar{E}_{1}\right)
$$


Заметим, что $\bar{F} \cap \bar{E}_{1}$ есть некратный неприводимьй слой эллиптического расслоения $\left.f\right|_{E_{1}}: \bar{E}_{1} \rightarrow \mathbb{P}^{1}$ и поверхность $\bar{E}_{1}$ имеет канонические особенности, поскольку мы можем считать линейную систему $|\bar{E}|$ свободной. Как легко подсчитать непосредственно, лог-пара $(* * *)$ будет лог-терминальной, если $\gamma<1,5 / 6,3 / 4,2 / 3,1 / 2,1 / 3,1 / 4,1 / 6$ и полный прообраз $\bar{F} \cap \bar{E}_{1}$ на минимальном разрешении поверхности $\bar{E}_{1}$ есть вырожденный слой эллиптической поверхности типа: стабильньй слой, $I I, I I I, I V, I_{b \geqslant 0}^{*}, I V^{*}$, $I I I^{*}, I I^{*}$ соответственно.

ЗАмЕчаниЕ 3. Если в теореме $1 I=1$ и $X$ имеет терминальные особенности, то $H^{3} \leqslant 6$. Действительно, можно в доказательстве теоремы 2 положить $\gamma=5 /(6+\varepsilon)$ $(0<\varepsilon \ll 1)$.

ЗАмЕчАниЕ 4. Если в теореме $1=1$ и $H^{3} \geqslant 48$, то возможны следуюшие варианты:

1)

2) линейная система $|H|$ задает двулистное накрытие многообразия минимальной степени ( $X$ гиперэллиптично).

Это следует из теоремы 2 и свойств подвижных линейных систем на поверхностях типа $K 3$ (см. [2] и [8]).

\section{3. $X$ - гиперэллиптично или тригонально.}

Лемма 1 (М. Рид). Пусть

$$
V \cong \operatorname{Proj}\left(\bigoplus_{i=1}^{m} \mathcal{O}_{\mathbb{P}^{1}}\left(d_{i}\right)\right) \quad u \quad Y_{j} \cong \operatorname{Proj}\left(\bigoplus_{i=j}^{m} \mathcal{O}_{\mathbb{P}^{1}}\left(d_{i}\right)\right)
$$

где $d_{1} \geqslant \cdots \geqslant d_{m}, d_{1}>d_{m} u m \geqslant j>1$. Отождествим $Y_{j}$ с подмногообразием в $V$, при вложсении определяемом естественной проекиией $\bigoplus_{i=1}^{m} \mathcal{O}_{\mathbb{P}}\left(d_{i}\right) \rightarrow$ $\bigoplus_{i=j}^{m} \mathcal{O}_{\mathbb{P}^{1}}\left(d_{i}\right)$. Пусть $s \in H^{0}\left(\mathcal{O}_{V / \mathbb{P}^{1}}(a) \otimes f^{*}\left(\mathcal{O}_{\mathbb{P}^{1}}(b)\right)\right)$, где $f: V \rightarrow \mathbb{P}^{1}$ естественная проекиия, а и $b$ иелье числа. Тогда $s$ имеет на $Y_{j}$ нуль порядка не меньше $q в$ том и только том случае, если выполняется следующее неравенство:

$$
\operatorname{ad}_{j}+b+\left(d_{1}-d_{j}\right)(q-1)<0 .
$$

ДоКАЗАТЕЛЬСТво. См. [2].

Лемма 2. Пусть в теореме $1 \quad I=1$ и $X$ гиперэллиптично (см. замечание 4). Тогда $H^{3} \leqslant 16$.

ДокаЗАТЕЛЬСтво (В гладком случае см. [2]). Легко видеть, что $\varphi_{|H|}(X)$ есть многообразие "минимальной степени", и, учитывая необходимое неравенство, можно считать, что $\varphi_{|H|}(X) \cong \varphi_{\left|\mathcal{O}_{V / \mathbb{P}}{ }^{(1)}\right|}(V)$, где $V \cong \operatorname{Proj}\left(\bigoplus_{i=1}^{3} \mathcal{O}_{\mathbb{P}^{1}}\left(d_{i}\right)\right)$ и $d_{1} \geqslant d_{2} \geqslant d_{3} \geqslant 0$.

Если $d_{1}=d_{2}=d_{3}$, то $H^{3} \leqslant 16$. Следовательно, можно считать, что $d_{1}>d_{3}$. Если $\varphi_{|H|}(X)$ особо в коразмерности 2 , то [11] влечет $H^{3} \leqslant 8$. Следовательно, можно считать, что $d_{2} \neq 0$. Заметим, что если $\varphi_{|H|}(X)$ особо, то $d_{3}=0$ и в обозначениях леммы $1 \varphi_{\left|\mathcal{O}_{V / \mathbb{P}^{1}}(1)\right|}$ стягивает кривую $Y_{3}$. 
Пусть $D$ есть собственный прообраз на $V$ дивизора ветвления двулистного накрытия $\varphi_{|H|}$. В обозначениях леммы 1

$$
D \sim \mathcal{O}_{V / \mathbb{P}^{1}}(4)-f^{*}\left(\mathcal{O}_{\mathbb{P}^{1}}\left(2\left(d_{1}+d_{2}+d_{3}-2\right)\right)\right)
$$

Рассматривая нормализацию расслоенного произведения $X \times_{\varphi_{|H|}(X)} V$, легко видеть, что $D$ содержит $Y_{3}$ с кратностью не больше 2 . Учитывая приведенность $D$, видим, что $D$ содержит $Y_{2}$ с кратностью не больше 1. Из леммы 1 следуют неравенства:

$$
\begin{array}{r}
d_{2}-d_{1}-2 d_{3}+4 \geqslant 0 \\
4-2 d_{2} \geqslant 0 .
\end{array}
$$

Откуда $H^{3}=2\left(d_{1}+d_{2}+d_{3}\right) \leqslant 16$.

ЛЕмма 3. Пусть в теореме $1 I=1$ и $X$ тригонально, т.е. Н очень обилен $u$ общий әлемент семейства кривых $\left\{H^{2}\right\}$ есть неприводимая гладкая тригональная кривая. Тогда $H^{3} \leqslant 54$.

ДокАЗАТЕЛЬСтво. Отождествим $X$ с его антиканоническим образом. Как и в случае гладкого $X$ (см. [2]), можно показать, что пересечение квадрик в $\mathbb{P}^{H^{3} / 2+2}$, содержащих $X$, есть $W \cong \varphi_{\left|\mathcal{O}_{V / \mathbb{P}}(1)\right|}(V)$, где $V \cong \operatorname{Proj}\left(\bigoplus_{i=1}^{4} \mathcal{O}_{\mathbb{P}^{1}}\left(d_{i}\right)\right)$ и $d_{1} \geqslant d_{2} \geqslant d_{3} \geqslant d_{4} \geqslant 0$.

Если $d_{1}=d_{2}=d_{3}=d_{4}$, то $H^{3} \leqslant 18$. Следовательно, можно считать, что $d_{1}>d_{4}$. Пусть $Z=\operatorname{Sing}(W) \cong \mathbb{P}^{k}(k=0,1,2)$, тогда $W$ есть конус с вершиной $Z$. Аналогично с гладким случаем $Z \cap X \subset \operatorname{Sing}(X)$. Заметим, что если $\operatorname{dim}(Z)=2$, то $\operatorname{dim}(Z \cap X)=1$, $d_{2}=d_{3}=d_{4}=0$ и в обозначениях леммы $1 \varphi_{\left|\mathcal{O}_{V / \mathbb{P}}(1)\right|}$ стягивает $Y_{2}$ на $Z$ и в общей точке $Z$ является раздутием.

Пусть $\bar{X}=\varphi_{\left|\mathcal{O}_{V / \mathbb{P}}(1)\right|}^{-1}(X)$. Учитьвая формулу суб-присоединения (см. [1]) и гладкость $V$, получаем что $\bar{X}$ имеет канонические особенности и в обозначениях леммы 1

$$
\bar{X} \sim \mathcal{O}_{V / \mathbb{P}^{1}}(3)+f^{*}\left(\mathcal{O}_{\mathbb{P}^{1}}\left(2-d_{1}-d_{2}-d_{3}-d_{4}\right)\right)
$$

$Y_{2}$ не принадлежит $\bar{X}$ в силу неприводимости $\bar{X} . \bar{X}$ содержит $Y_{3}$ с кратностью не больше 1, а $Y_{4}$ с кратностью не больше 2. Из леммы 1 следуют неравенства:

$$
\begin{array}{r}
2 d_{2}-d_{1}-d_{3}-d_{4}+2 \geqslant 0 \\
d_{3}-d_{2}+2 \geqslant 0 \\
2-d_{2}-d_{3}+d_{1} \geqslant 0
\end{array}
$$

Откуда $H^{3}=2+2\left(d_{1}+d_{2}+d_{3}+d_{4}\right) \leqslant 54$.

\section{4. $X$ заметается "прямыми".}

Лемма 4. Пусть $x$ есть замкнутая гладкая точка $k$-мерного многообразия $X$, $H$ есть обильный дивизор Картье на $X$. Предположим, что общую точку $v \in X$ соединяет с $x$ неприводимая кривая $C_{v}$ такая, что $H C_{v} \leqslant d$. Тогда $H^{k} \leqslant d^{k}$. 
ДокАЗАТЕЛЬСтво. $h^{0}\left(\mathcal{O}_{X}(m H)\right)=m^{k} H^{k} / k !+O\left(m^{k-1}\right)$ для $m \in \mathbb{N}_{\gg 0}$. Если $H^{k}>d^{k}$, то для $m \gg 0$ существует дивизор $D \in H^{0}\left(\mathcal{O}_{X}(m H)\right)$, имеюший в точке $x$ кратность не меньше $(m d+1)$. Следовательно, $D$ содержит все кривые $C_{v}$, что невозможно.

Лемма 5. Если в теореме $1 \quad I=1$ и через общую точку $v \in X$ проходит неприводимая кривая $C_{v}$ такая, что $H C_{v}=1$, то $H^{3} \leqslant 46$.

ДокАЗАтЕльство. Если $\mathrm{Bs}|H| \neq \varnothing$, то теорема 2 влечет $H^{3} \leqslant 46$. Если $\mathrm{Bs}|H|=\varnothing$, то пусть $\{C\}$ есть семейство неприводимых приведенных кривых таких, что $H C=1$ и через общую точку $X$ проходит кривая из $\{C\}$. Из замечания 4 следует, что $C \cong \mathbb{P}^{1}$.

Рассмотрим RC-расслоение (см. [12], [13]) $\varphi: X \rightarrow W$, ассоциированное с $\{C\}$.

Если $\operatorname{dim}(W)=0$, то две общие точки $X$ могут быть соединены цепочкой не более чем трех кривых из $\{C\}$, которые можно склеить и получить новое семейство $\left\{C^{\prime}\right\}$ такое, что две обшие точки $X$ могут быть соединены одной кривой из семейства $\left\{C^{\prime}\right\}$ (см. [13]). Применение леммы 4 к $\left\{C^{\prime}\right\}$ дает неравенство $H^{3} \leqslant 26$ ( $H^{3}$ четно).

Рассмотрим коммутативную диаграмму:

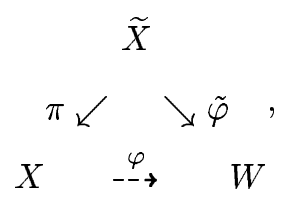

где $\widetilde{X}$ это гладкое многообразие, $\pi$ бирационален и $\tilde{\varphi}-$ морфизм.

Если $\operatorname{dim}(W)=1$, то пусть $S$ есть общий слой морфизма $\tilde{\varphi}$, а $C$ есть общая кривая из $\{C\}$. Семейство кривых $\left\{\pi^{-1}(C)\right\}$ определяет на $S$ семейство кривых $\left\{C_{S}\right\} \in S$. Общая кривая $C_{S}$ из $\left\{C_{S}\right\}$ неприводимая, гладкая, рациональная, $-K_{S} C_{S} \leqslant 1$ и $C_{S}^{2}>0$, что противоречит формуле присоединения.

Если $\operatorname{dim}(W)=2$, то пусть $C$ есть общая кривая из $\{C\} ; \pi^{-1}(C)$ есть общий слой морфизма $\tilde{\varphi}$ и $-K_{\tilde{X}} \pi^{-1}(C) \leqslant 1$, что противоречит $-K_{\tilde{X}} \pi^{-1}(C)=2$, поскольку общий слой $\tilde{\varphi}$ есть $\mathbb{P}^{1}$.

\section{5. Двойная проекция из общей точки $X$.}

Лемма 6. Пусть в теореме $1 \quad I=1, H^{3} \geqslant 56 u \pi: \widehat{X} \rightarrow X$ есть раздутие достаточно общей точки $v \in X$ с исключительным дивизором Е. Тогда дивизор $\pi^{*}(H)-2 E$ численно әффективен и оббемен.

ДокАЗАТЕЛЬСтво. Достаточно доказать, что $\pi^{*}(H)-2 E$ численно эффективен. Из замечания 4 и леммы 2 следует, что $H$ очень обилен. Отождествим $X$ с его антиканоническим образом. Если $\left(\pi^{*}(H)-2 E\right) C<0$ для кривой $C \in \widehat{X}$, то $v \in \pi(C) \in T_{v}(X) \cap X$, где $T_{v}(X)$ есть касательное пространство к $X$ в точке $v$. Из леммы 3 , теоремы Нетера-Энриквеса-Петри (см. [14]) и [2] следует, что $X$ высекается квадриками. Последнее влечет, что $C$ есть прямая на $X$ проходящая через точку $v$, но по лемме 5 это невозможно.

ДоКАЗАТЕЛЬСТво тЕОРЕМЫ 1 . По замечанию 1 достаточно доказать теорему 1 при условии $I=1$. Пусть $\pi: \widetilde{X} \rightarrow X$ есть раздутие общей точки $v \in X$. Если $H^{3} \geqslant 56$, то по лемме 6 дивизор $K_{\tilde{X}} \sim \pi^{*}(H)-2 E$ будет численно эффективен и объемен. По теореме о свободе от базисных точек (см. [1]) существует $N \in \mathbb{N}$ такое, что для $n \in \mathbb{N}_{\geqslant N}$ линейная 
система $\left|n\left(\pi^{*}(H)-2 E\right)\right|$ свободна. Следовательно, для $n \gg 0$ существует морфизм $\varphi: \widetilde{X} \rightarrow X^{\prime}$ такой, что $X^{\prime}$ имеет канонические особенности,

$$
\pi^{*}(H)-2 E=\varphi^{*}\left(H^{\prime}\right), \quad-K_{X^{\prime}} \sim H^{\prime} \text { и } H^{\prime 3}=H^{3}-8,
$$

где $H^{\prime}$ это обильньй дивизор Картье.

В условиях нашей теоремы можно повторить данную перестройку 17 раз. Таким образом, можно считать, что $\pi$ есть раздутие 17 точек $X$ в общем положении.

Обший дивизор $\widetilde{D} \in\left|-K_{\widetilde{X}}\right|$ приведен, неприводим, имеет лишь канонические особенности и есть поверхность типа $K 3$ (см. [15]). Поскольку дивизор $-K_{\tilde{X}} \pi$-обилен, то морфизм $\pi$ определяет на $\widetilde{D}$ стяпивание 17 кривых, которые попарно не пересекаются, что невозможно по [16].

\section{СПИСОК ЦИТИРОВАННОЙ ЛИТЕРАТУРЫ}

[1] Kawamata Y., Matsuda K., Matsuki K. Introduction to the minimal model problem // Adv. Stud. Pure Math. 1987. V. 10. P. 383-360.

[2] Исковских В.А. Антиканонические модели трехмерных алгебраических многообразий // Современные проблемы математики. 1979. Т. 12. С. 59-157.

[3] Чельцов И. А. Трехмерные многообразия, обладающие дивизором с численно тривиальным каноническим классом // УМН. 1996. Т. 1. С. 177-178.

[4] Sano T. On classifications of non-Gorenstein $\mathbb{Q}$-Fano 3-folds of Fano index 1 // J. Math. Soc. Japan. 1995. V. 47. № 2. P. 369-380.

[5] Namikawa Y. Smoothing Fano 3-folds. Preprint, 1995.

[6] Fano G. Sulle varieta algebriche a tre dimensioni a curve-sezioni canoniche // Mem. Acc. It. 1937. V. 8. P. 813-818.

[7] Fano G. Sulle varrieta algebriche a tre dimensionile le cui sezioni iperpiane sono superfici di genere zero e bigenere uno // Mem. Soc. It. d. Scienze (detta dei XL). 1938. V. 24. P. 44-66.

[8] Shin K.-H. 3-dimensional Fano varieties with canonical singularities // Tokyo J. Math. 1989. V. 12. P. $375-385$.

[9] Kawamata Y. Crepant blowing-up of 3-dimensional canonical singularities and its application to degenerations of surfaces // Ann. of Math. 1988. V. 127. P. 93-163.

[10] Kawamata Y. On Fujita's freeness conjecture for 3-folds and 4-folds. Preprint, 1996.

[11] Saint-Donat B. Projective models of $K-3$ surfaces // Amer. J. Math. 1974. V. 96. № 4. P. 602-639.

[12] Kollár J., Miyaoka Y., Mori S. Rational connectedness and boundedness of Fano manifolds // J. Differential Geom. 1992. V. 36. № 3. P. 765-779.

[13] Kollár J., Miyaoka Y., Mori S. Rationally connected varieties // J. Algebraic Geom. 1992. V. 1. P. 429-448.

[14] Шокуров В.В. Теорема Нетера-Энриквеса о канонических кривых // Матем. сб. 1971. T. 86. № 3. C. 367-408.

[15] Reid M. Projective morphism according to Kawamata. Preprint. University of Warwick, 1983.

[16] Никулин В. В. О Куммеровых поверхностях // Изв. АН СССР. Сер. матем. 1975. Т. 39. C. $278-293$.

Математический институт им. В. А. Стеклова РАН

Поступило 24.04 .97

Исправленный вариант 06.10 .98 